\title{
8-Amino guanine accelerates tetramolecular G-quadruplex formation $\nmid \ddagger$
}

\author{
Julien Gros, ${ }^{a}$ Anna Aviñó, ${ }^{b}$ Jaime Lopez de la Osa, ${ }^{c}$ Carlos González, ${ }^{c}$ Laurent Lacroix, ${ }^{a}$ \\ Alberto Pérez, ${ }^{d}$ Modesto Orozco, ${ }^{d e}$ Ramon Eritja ${ }^{* b}$ and Jean-Louis Mergny ${ }^{* a}$
}

\author{
Received (in Cambridge, UK) 22nd January 2008, Accepted 11th March 2008 \\ First published as an Advance Article on the web 15th April 2008 \\ DOI: $10.1039 / b 801221 \mathrm{k}$
}

We demonstrate here that 8-amino guanine (R) strongly accelerates quadruplex formation, which makes this nucleobase the most attractive replacement for guanine in the context of tetramolecular parallel quadruplexes.

G-Quadruplexes are a family of four-stranded DNA structures stabilized by guanine quartets. These structures have a low tolerance for non-guanine quartets which are rather formed by virtue of the docking platform provided by neighboring G-quartets. Few exceptions have been found. We demonstrate here that 8-amino guanine (R, Fig. 1) strongly accelerates quadruplex formation, which makes this nucleobase the most attractive replacement for guanine in the context of parallel quadruplexes.

We recently analysed the impact of a number of base analogs on parallel-stranded, tetramolecular quadruplex formation. ${ }^{1}$ Most guanine substitutions are tolerated at best and are only formed thanks to the docking platform provided by neighboring G-quartets. Exceptions to this "guanine only" rule are guanine analogs modified at position $8 .{ }^{1-3}$ Given the position of that substituent, which does not perturb the cyclic arrangement of H-bonds but favors the syn sugar base conformation, we wondered what would be the effect of other substituents at this position. 8-Amino guanine was previously found to stabilize triplexes ${ }^{4}$ but destabilize quadruplexes ${ }^{5}$ when inserted in the position 1 of an intramolecular quad-

\footnotetext{
${ }^{a}$ Muséum National d'Histoire Naturelle (MNHN) USM 503, INSERM U565, CRNS UMR 5153; 43 rue Cuvier CP26, F-75231 Paris Cedex05, France.E-mail: mergny@mnhn.fr;

Fax: + 331407937 05: Tel: + 33140793689

${ }^{b}$ IRB Barcelona, CIBER-BBN, CSIC Josep Samitier 1-5, 08028

Barcelona, Spain. E-mail: recgma@cid.csic.es;

Fax: + 34-93-2045904; Tel: + 34-93-4039942

${ }^{c}$ Instituto de Química Física Rocasolano, CSIC, C/Serrano, 119, 28006 Madrid, Spain

${ }^{d}$ Joint IRB-BSC program on Computational Biology, Institut de Recerca Biomèdica, Parc Científic de Barcelona, Josep Samitier 1-5, 08028 Barcelona, Spain, and Barcelona Supercomputing Center, Jordi Girona 29, 08034 Barcelona, Spain

${ }^{e}$ Departament de Bioquimica, Facultat de Biologia, Universitat de Barcelona, Avgda Diagonal 647, 08028 Barcelona, Spain

$\dagger$ Experimental methods: Spectroscopy. Several biophysical methods have been used to follow quadruplex formation. As they have all been previously described, ${ }^{7}$ their complete description appear as supplementary information. Theoretical calculations. Quantum mechanical and molecular dynamics simulations have been used to study both the syn/anti equilibrium of guanine and 8-amino guanine in aqueous solution and the impact of the guanine to 8-amino guanine substitution in the structure and stability of parallel quadruplexes. Full details of the theoretical methods used are included as supplementary information.

$\ddagger$ Electronic supplementary information (ESI) available: Complete
} protocols, 8 figures and 2 tables. See DOI: 10.1039/b801221k ruplex. Following suggestions derived from preliminary theoretical calculations we decided to evaluate the effect of 8 -amino guanine when inserted in the internal region of a parallel G-quadruplex.

Evidence for quadruplex formation with 8-amino G-containing sequences. Electrophoresis was used to evidence quadruplex formation (Fig. 2A). In native acrylamide gels, migration of the unmodified G4-DNA structure is characterized by a slight migration delay as compared to single-strands. By replacing each $\mathrm{G}$ in $\mathrm{d}\left(\mathrm{TG}_{4} \mathrm{~T}\right)$ or $\mathrm{d}\left(\mathrm{TG}_{5} \mathrm{~T}\right)$ sequences with $\mathbf{R}$, we observed different migrations for structured and singlestranded forms.

Circular dichroism (CD) provided additional evidence for modified G4-DNA formation. CD spectra of all sequences are characterised by a negative peak at $242 \mathrm{~nm}$, and a positive peak at $263 \mathrm{~nm}$ (Fig. 2B), referenced as "type 1 spectrum" in the literature. Isothermal difference spectra (IDS) of both modified and unmodified sequences were also characteristic for G4-DNA formation with two positive peaks at 240 and $273 \mathrm{~nm}$, and two negative peaks at 260 and $295 \mathrm{~nm}$. The shapes of these IDS confirm that all sequences form quadruplexes.

NMR spectra of all unmodified and modified sequences were consistent with quadruplex formation (see ESI). $\ddagger$ In all cases, guanine imino protons were observed around 11.0-11.5 ppm. These signals were still observed at very high temperatures $\left(>75{ }^{\circ} \mathrm{C}\right)$. In general, the modified oligonucleotides exhibited broader signals than the unmodified ones at low temperature $\left(5^{\circ} \mathrm{C}\right)$. These signals became narrower at higher temperatures. The spectra of $\left[\mathrm{d}(\mathrm{TGGRGGT)}]_{4}\right.$ was similar to unmodified [d(TGGGGGT) $]_{4}$ and the non-exchangeable resonances could be assigned. On the other hand, there were significant changes in the NMR spectra of [d(TRGGGGT) $]_{4}$

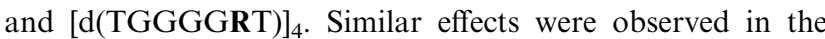
spectra of $[\mathrm{d}(\mathrm{TRGGGT})]_{4}$ and $[\mathrm{d}(\mathrm{TGGGRT})]_{4}$. The exceeding
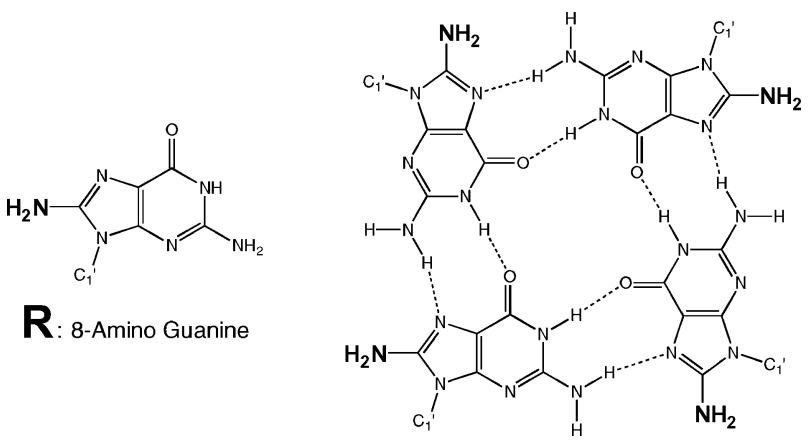

Fig. 1 8-Amino guanine ( $\mathbf{R}$, left) and schematic representation of the putative "R-tetrad" formed by the auto association of $4 \mathbf{R}$ (right). 


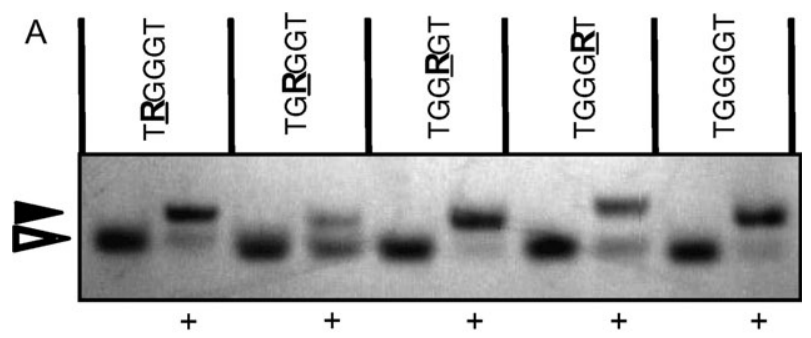

B

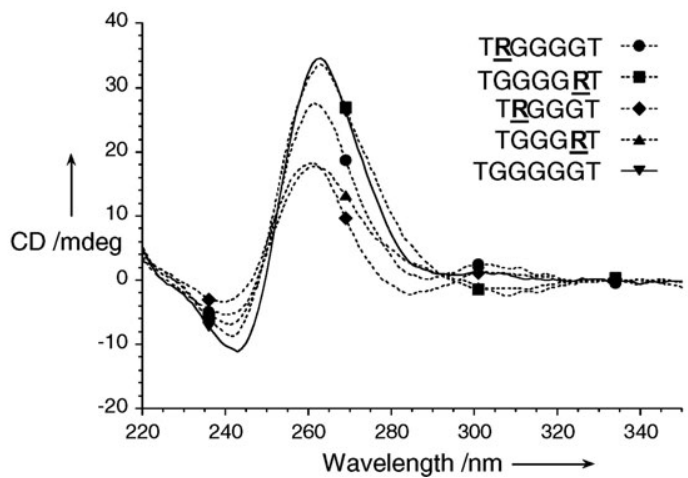

Fig. 2 (A) Tetramolecular quadruplex formation is correlated to the apparition of slow-migrating bands (full triangle) as compared to migration of single-strands (open triangle). Results obtained in sodium conditions with $\mathrm{TG}_{4} \mathrm{~T}$ sequences bearing an $\mathbf{R}$ at positions 1, 2, 3 or 4 . Samples "+ " were preincubated during $72 \mathrm{~h}$ at $4{ }^{\circ} \mathrm{C}$ at $100 \mu \mathrm{M}$ strand concentration in a $10 \mathrm{mM}$ lithium cacodylate $\mathrm{pH} 7.2$ buffer containing $110 \mathrm{mM} \mathrm{NaCl}$. In parallel, samples "-" were incubated under the same conditions omitting cations. (B) CD spectra in $10 \mathrm{mM}$ lithium cacodylate pH 7.2 buffer containing $110 \mathrm{mM} \mathrm{NH}_{4} \mathrm{Cl}$.

number of signals, probably due to the presence of minor species, make difficult the assignment of the NMR spectra of these modified quadruplexes. As in previously studied structures containing 8-amino guanines, the signals of the amino protons at position 8 were not observed in the NMR spectra. As a consequence, the conformation of their glycosidic angles could not be determined but, in all sequences, the medium or weak intensities of the $\mathrm{H} 8-\mathrm{H} 1^{\prime}$ NOEs indicated that the glycosidic angles of the unmodified guanines were anti. NMR data were consistent with quantum mechanical calculations which suggested no difference in the syn/anti free energy difference between guanine and 8-amino guanine $\left(\Delta \Delta \mathrm{G}_{\text {syn/anti- }}\right.$ $(\mathrm{R}-\mathrm{G})=0.1 \mathrm{kcal} \mathrm{mol}^{-1}$; see supplementary Methods $\ddagger$. There also was a clear agreement between NMR and MD simulations, which strongly suggest that minimum changes were due to the presence of 8-amino guanine in the quadruplex.

Association of G4 DNA. Association of a G4-DNA, which may be followed by absorbance, is generally slow and depends on temperature, oligonucleotide concentration, and nature and concentration of the monovalent cation. ${ }^{1,6-9}$ Replacement of a single $\mathrm{G}$ by 8 -amino guanine $(\mathbf{R})$ at various positions of $\mathrm{d}\left(\mathrm{TG}_{4} \mathrm{~T}\right)$ or $\mathrm{d}\left(\mathrm{TG}_{5} \mathrm{~T}\right)$ has been tested in this study and results are presented in Table 1.

In summary:

(1) As for both unmodified sequences, association rate of any modified sequence depends on the nature of the cation type: $k_{\text {on }}\left(\mathrm{K}^{+}\right)>k_{\text {on }}\left(\mathrm{Na}^{+}\right)>k_{\text {on }}\left(\mathrm{NH}_{4}{ }^{+}\right)$and its concentration $k_{\mathrm{on}}\left(\mathrm{K}^{+} 0.11 \mathrm{M}\right)>k_{\mathrm{on}}\left(\mathrm{K}^{+} 0.05 \mathrm{M}\right)>k_{\mathrm{on}}\left(\mathrm{K}^{+} 0.01 \mathrm{M}\right)$.
Table $1 k_{\text {on }}$ and $T_{1 / 2}$ for $\mathrm{TG}_{5} \mathrm{~T}$ and $\mathrm{TG}_{4} \mathrm{~T}$ variants ${ }^{a}$

\begin{tabular}{|c|c|c|c|c|c|c|}
\hline \multirow{2}{*}{$\begin{array}{l}\text { Sequences } \\
5^{\prime} \Rightarrow 3^{\prime}\end{array}$} & \multicolumn{3}{|c|}{$k_{\mathrm{on}} / \mathrm{M}^{-3} \mathrm{~s}^{-1 b}$} & \multicolumn{3}{|c|}{$\underline{T_{1 / 2}\left({ }^{\circ} \mathrm{C}\right)^{c}}$} \\
\hline & $\mathrm{K}^{+}$ & $\mathrm{Na}^{+}$ & $\mathrm{NH}_{4}{ }^{+}$ & $\mathrm{K}^{+}$ & $\mathrm{Na}^{+}$ & $\mathrm{NH}_{4}{ }^{+}$ \\
\hline TGGGGGT & $2.310^{12}$ & $9.810^{9}$ & $1.110^{9}$ & $>90$ & $>90$ & $>90$ \\
\hline TXGGGGT & $1.210^{13}$ & $1.110^{11}$ & $1.310^{10}$ & $>90$ & $>90$ & $>90$ \\
\hline TRGGGGT & Fast & Fast & $4.310^{10}$ & $>90$ & $>90$ & $>90$ \\
\hline TGGRGGT & $6.910^{10}$ & $8.710^{8}$ & $\mathrm{Nd}$ & $\mathrm{Nd}$ & $\mathrm{Nd}$ & $\mathrm{Nd}$ \\
\hline TGGGGRT & $3.910^{11}$ & $6.510^{9}$ & $4.510^{8}$ & $>90$ & $>90$ & $>90$ \\
\hline TGGGGXT & $2.510^{10}$ & $1.910^{8}$ & $5.410^{7}$ & $>90$ & $>90$ & $>90$ \\
\hline TGGGGT & $1.810^{10}$ & $3.510^{8}$ & $1.210^{8}$ & $>90$ & 57.3 & 74.7 \\
\hline TRGGGT & $4.410^{11}$ & $1.710^{10}$ & $2.110^{9}$ & $>90$ & 45.8 & 76.6 \\
\hline TGGGRT & $210^{10}$ & $3.610^{8}$ & $1.810^{7}$ & $>90$ & 38.2 & 58.7 \\
\hline
\end{tabular}

${ }^{a} \mathbf{X}$ and $\mathbf{R}$ correspond to 8-bromo-guanine and 8-amino guanine, respectively.

${ }^{b}$ The association rate $\left(\right.$ at $4{ }^{\circ} \mathrm{C}$ ) is provided in $\mathrm{M}^{-3} \mathrm{~s}^{-1}$ (assuming fourth order association kinetics ${ }^{1,6}$; see a recent discussion in ref. 10) in a $10 \mathrm{mM}$ lithium cacodylate $\mathrm{pH} 7.2$ buffer supplemented with $110 \mathrm{mM} \mathrm{KCl}, \mathrm{NaCl}$ or $\mathrm{LiCl} . \mathrm{Nd}$ : not done. Fast: formation of the quadruplex is too fast at $110 \mathrm{mM} \mathrm{KCl}$ to be followed under these ionic conditions even at the lowest practical strand concentration. At $10 \mathrm{mM} \mathrm{KCl}$, association of TRGGGGT is 7-fold and 63-fold faster than TXGGGGT and TGGGGGT, respectively (not shown). ${ }^{c} T_{1 / 2}$ in ${ }^{\circ} \mathrm{C}$ correspond to non-reversible apparent melting temperatures and depend on the temperature gradient $\left(0.5{ }^{\circ} \mathrm{C} \min ^{-1}\right.$ here $)$. $>90$ : no significant denaturation occurred during the melting experiment.

(2) $\mathrm{d}\left(\mathrm{TG}_{5} \mathrm{~T}\right)$ always leads to faster associations than $\mathrm{d}\left(\mathrm{TG}_{4} \mathrm{~T}\right)$, and similar results are found for modified sequences. For example, $k_{\mathrm{on}}\left([\mathrm{d}(\mathrm{TRGGGGT})]_{4}\right)>k_{\mathrm{on}}\left([\mathrm{d}(\mathrm{TRGGGT})]_{4}\right)$.

(3) Replacement at the $5^{\prime}$ end is more favorable than at the $3^{\prime}$ end, while insertion of $\mathbf{R}$ at central position hinders the association: $k_{\text {on }}\left([\mathrm{d}(\text { TRGGGGT })]_{4}\right)>k_{\text {on }}\left([\mathrm{d}(\text { TGGGGRT })]_{4}\right)$ $>k_{\text {on }}\left([\mathrm{d}(\mathrm{TGGRGGT})]_{4}\right)$. A similar $5^{\prime} / 3^{\prime}$ asymmetry was found for the shorter sequences.

(4) When considering the $5^{\prime}$ position, $\mathbf{R}$ leads to the fastest association kinetics among all tested bases, including guanine and 8 -bromo guanine $(\mathbf{X})$. Fig. 3 presents the kinetics of formation of the control sequence compared to the modified oligonucleotides. It illustrates the increased rate observed with the d(TRGGGGT) sequence.

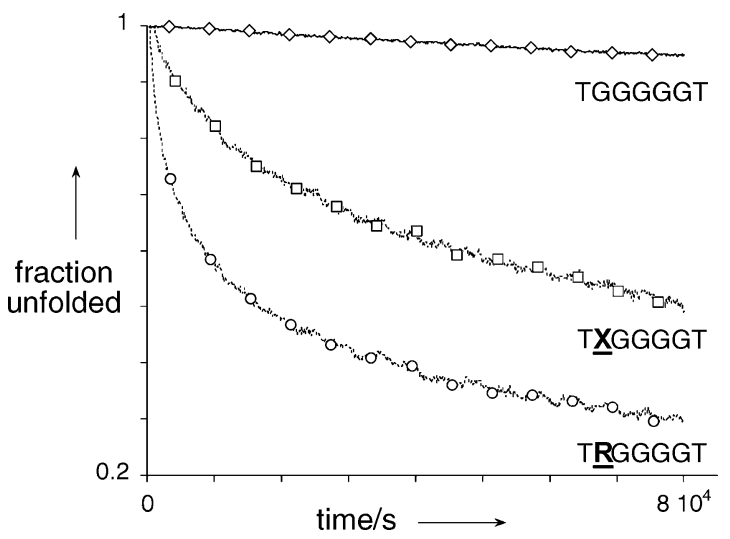

Fig. 3 Accelerated quadruplex formation with 8-amino guanine. Acceleration of the association by replacing the first $\mathrm{G}$ at $5^{\prime}$ end on $\mathrm{TG}_{5} \mathrm{~T}$ by 8 -bromo guanine $(\mathbf{X})$ or 8 -amino guanine $(\mathbf{R})$. The three oligonucleotides have been used at the same concentration: $(8.7 \pm$ $0.15) \mu \mathrm{M}$. Association was followed by absorbance at $(3.9 \pm 0.3){ }^{\circ} \mathrm{C}$ in a $10 \mathrm{mM}$ lithium cacodylate $\mathrm{pH} 7.2$ buffer with $10 \mathrm{mM} \mathrm{KCl}+$ $100 \mathrm{mM} \mathrm{LiCl}$. 


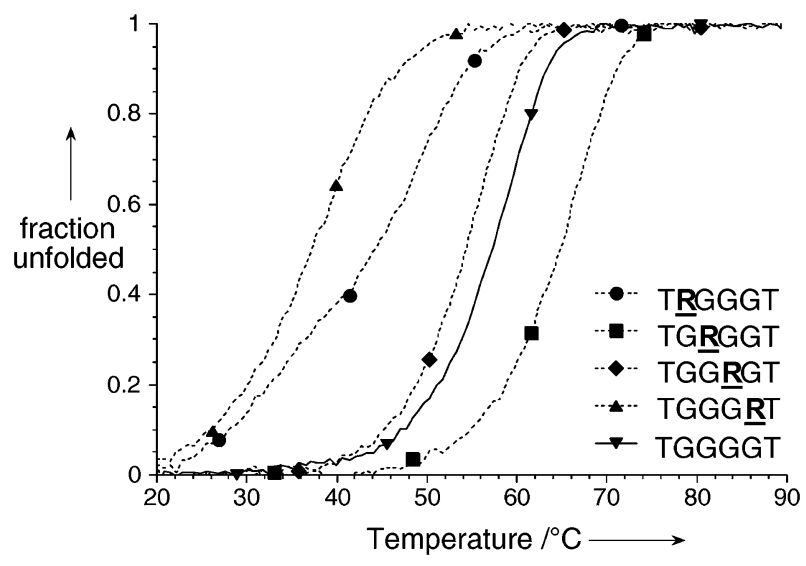

Fig. 4 Effect of $\mathbf{R}$ on $\mathrm{G} 4$ formation and thermal stability. Melting profiles of G4-DNA prepared in $\mathrm{NaCl} 110 \mathrm{mM}$, based on $\mathrm{TG}_{4} \mathrm{~T}$ sequences and modified at each positions with $\mathbf{R}$.

Thermal stability of quadruplexes containing 8-amino guanine. The thermal stability of preformed quadruplexes was analyzed by UV-absorbance spectroscopy. An example of a thermal experiment followed by UV absorbance spectroscopy is shown in Fig. 4. Results are presented in Table 1 and may be summarized as follows:

(1) Temperatures of half-dissociation $\left(T_{1 / 2}\right)$ measured depend on the heating rate and on the nature of the cation: $T_{1 / 2}\left(\mathrm{~K}^{+}\right)>T_{1 / 2}\left(\mathrm{NH}_{4}{ }^{+}\right)>T_{1 / 2}\left(\mathrm{Na}^{+}\right)$but weakly on its concentration.

(2) The $\mathbf{R}$ substitution has little or no detrimental effect on the thermal stability of the quadruplexes. Insertion of $\mathbf{R}$ at central positions in $\mathrm{d}\left(\mathrm{TG}_{4} \mathrm{~T}\right)$ sequence even leads to an increase in the apparent melting temperature: $T_{1 / 2}\left[\mathrm{~d}\left(\mathrm{TGRG}_{2} \mathrm{~T}\right)\right]_{4}$ $>T_{1 / 2}\left[\mathrm{~d}\left(\mathrm{TG}_{4} \mathrm{~T}\right)\right]_{4}>T_{1 / 2}\left[\mathrm{~d}\left(\mathrm{TG}_{2} \mathbf{R G T}\right)\right]_{4}$ in $\mathrm{NaCl}$.

The lack of destabilization (or even the gain of stability) of quadruplexes by 8-amino guanine insertion in the middle of the structure seems in disagreement with previous theoretical and experimental studies, which found destabilization of the structure when $\mathbf{R}$ replaced one $\mathrm{G}$ in a two-step intramolecular quadruplex. $^{5}$ In order to solve this apparent paradox, we performed $\mathrm{MD} / \mathrm{TI}$ calculations similar to those in ref. 5, but now considering none, one, or four $\mathrm{G}$ to $\mathbf{R}$ substitutions at the 2nd step of the $[\mathrm{d}(\mathrm{GGGG})]_{4}$ quadruplex (see supplementary Methods). $\ddagger$ Results showed an important gain of stability (around $2.4 \mathrm{kcal} \mathrm{mol}^{-1}$ ) when one guanine is substituted by one 8-amino guanine, the difference being enlarged to $9.5 \mathrm{kcal}$ $\mathrm{mol}^{-1}$ when the entire $2 \mathrm{nd}$ step is changed to 8 -amino guanines, in agreement with experimental measures and in opposition to what was found previously in the analysis of external
$\mathbf{G}$ to $\mathbf{R}$ changes. Theoretical calculations therefore support the marked sequence-dependent effect of the $G$ to $\mathbf{R}$ substitution and the fact that the introduction of the modified nucleotide in the interior of the G-quadruplex can stabilize the structure.

In order to gain an atomistic description of the reasons that can justify the gain in stability induced by 8 -amino guanine in the center of the quadruplex, we compared the hydrogen bond, stacking and ion-nucleobase interaction energies for parent quadruplexes and those that contained one or four 8-amino guanines at the 2nd position using our multi-nanosecond trajectories of the different structures. Results summarized in supplementary Table S1 clearly illustrate that the driving force for the stabilization of the quadruplex induced by the $G$ to $\mathbf{R}$ change is the gain in nucleobase-ion interaction energy, which originates from the better dipole orientation in $\mathbf{R}$ (see supplementary Fig. S7) $\ddagger$ As ion density is larger in internal steps (surrounded by two ions) than for the external ones, the preference of $\mathbf{R}$ for internal positions is easily explained. Furthermore, our calculations suggest an important dependence of the stabilization effect of $\mathbf{R}$ on the nature of the ion placed inside the central G-channel.

The 8-amino guanine replacement is not universal, as it is not equally favourable at all positions. Clearly more work is required to find truly universal guanine analogs.

This work was supported by ARC (\#3365 to J.L.M), and E.U. FP6 "MolCancerMed” (LSHC-CT-2004-502943) grants. We thank Elena Cubero (IRB, Barcelona) for preliminary $\mathrm{MD} / \mathrm{TI}$ simulations.

\section{Notes and references}

1. J. Gros, F. Rosu, S. Amrane, A. De Cian, V. Gabelica, L. Lacroix and J. L. Mergny, Nucleic Acids Res., 2007, 35, 3064-3075.

2. V. Gubala, J. E. Betancourt and J. M. Rivera, Org. Lett., 2004, 6, 4735-4738.

3. L. Petraccone, I. Duro, A. Randazzo, A. Virno, L. Mayol and C. Giancola, Nucleosides, Nucleotides Nucleic Acids, 2007, 26, 669-674.

4. R. Soliva, R. Guimil Garcia, J. R. Blas, R. Eritja, J. L. Asensio, C. Gonzalez, F. J. Luque and M. Orozco, Nucleic Acids Res., 2000, 28, 4531-4539.

5. J. Lopez de la Osa, C. Gonzalez, R. Gargallo, M. Rueda, E. Cubero, M. Orozco, A. Avino and R. Eritja, ChemBioChem, 2006, 7, 46-48.

6. J. R. Wyatt, P. W. Davis and S. M. Freier, Biochemistry, 1996, 35, 8002-8008.

7. J. L. Mergny, A. De Cian, A. Ghelab, B. Saccà and L. Lacroix, Nucleic Acids Res., 2005, 33, 81-94.

8. J. L. Mergny, A. De Cian, S. Amrane and M. Webba da Silva, Nucleic Acids Res., 2006, 34, 2386-2397.

9. A. De Cian and J. L. Mergny, Nucleic Acids Res., 2007, 35, 2483-2493.

10. C. Bardin and J. L. Leroy, Nucleic Acids Res., 2008, 36, 477-488. 